\title{
Interactive expert support for early stage full-year daylighting design: a user's perspective on Lightsolve
}

\author{
Authors: \\ Marilyne Andersen ${ }^{1,2}$ (Corresponding Author ${ }^{*}$ ) \\ Email: marilyne.andersen@epfl.ch \\ Jaime M.L. Gagne ${ }^{2,3}$ \\ Email: jaime@kgsbuildings.com
}

Siân Kleindienst ${ }^{2,3}$

Email: sian@kgsbuildings.com

${ }^{1}$ Interdisciplinary Laboratory of Performance-Integrated Design (LIPID), School of Architecture, Civil and Environmental Engineering (ENAC), Ecole Polytechnique Fédérale de Lausanne (EPFL), Switzerland

${ }^{2}$ Building Technology Program, Department of Architecture, Massachusetts Institute of Technology (MIT), USA

${ }^{3}$ Present address: KGS Buildings, Cambridge MA, USA

\begin{abstract}
:
Designing spaces that are able to balance illumination, glare and solar gains over a year is a real challenge, yet a problem faced every day by building designers. To assist them, a full year, climate-based daylighting simulation method, called Lightsolve, was developed, providing guided search based on the variation of daylight performance over the year by combining temporal performance visualization with spatial renderings. This paper focuses on the user's perspective for Lightsolve. After a summary of its foundational concepts, it discusses the results of several pilot and more formal user studies conducted in educational contexts. As a core element of the paper, the method and results of an original, design-oriented user study on Lightsolve's expert system are discussed. It was conducted to determine how well its decision-making algorithm would work when independent human interactions were included. It demonstrated that the expert system is generally successful as a performance-driven design tool respectful of the non-deterministic nature of the design process itself, and as a method for educating designers to improve daylighting performance.
\end{abstract}

Keywords: daylighting, goal-based design support, expert system, climate-based modeling, guided search, user interactivity

Word count: 8619

\section{Highlights:}

- Comprehensive, goal-based design support focused on daylight dynamics

- Original user study was designed to integrate ill-defined nature of design process

- User studies confirmed tool potential for interactivity in early stages of design

- Expert system shown successful in educating designers about daylighting performance

\footnotetext{
* Address for correspondence: EPFL ENAC IA LIPID, Building BP 2229, Station 16, CH - 1015 Lausanne. Tel: +41 216930882
} 


\section{Introduction}

Daylighting design is both highly relevant to cutting edge societal issues such as energy conservation, sustainability and health, and highly sensitive to careful planning and control [1-4]. Its benefits, however, can only be effective if one also carefully accounts for our visual needs and comfort criteria. The main challenge resides in the reconciliation of the many factors influencing how daylight and sunlight each interact with the built environment and in the great variations they show in intensity and distribution depending on location, weather and time.

Today, simulation tools have become the dominant form of design support [5-6] but due to the large number of parameters involved and the need for detailed, climate-based analyses to be realistic about daylighting potential [7-8], evaluating annual daylighting performance of a schematic building project interactively and comprehensively remains challenging.

In fact, daylighting is a field where strictly defined numerical boundaries do not apply: there is a vast range of parameters and values that are relevant to "good" daylighting, which makes absolute performance targets of questionable relevance. This is probably one of the reasons why very few papers have tried to address the problem of daylighting optimization [9-11] except for simplified guidance derived from knowledge-based systems [12-14].

The simulation platform and associated expert system considered in the present paper differs from these previous efforts in that it allows a comprehensive understanding of daylighting and offers user interactivity regarding design choices. Daylighting performance is evaluated as a function of daily and seasonal variations against the user's own performance objectives and to his or her chosen areas of interest. Details about this approach have been published in earlier papers [14-17] and will only be briefly summarized in section 2 .

With any new method, it is critical to determine if the intended audience finds it more useful than existing methods. After an overview of the main components of Lightsolve as a whole in section 2, this paper will present the results of a series of user studies conducted with designers and students. Two preliminary user surveys, presented in section 3, were given to both test the usefulness and intuitive nature of Lightsolve's temporal approach, and to judge how intuitive 
temporal data was to the inexperienced architect. At a later stage of tool development, a threephase user study specifically addressing the design support potential of Lightsolve - combining design by hand and use of analysis tools - was given, with a dedicated focus on its expert system.

Several important results were expected from this study. The first is an assessment of the ability of the expert system to find designs with improved daylighting performance when a human user is allowed to interact with it in an independent way. The second is an evaluation of the expert system process as a method for improving a designer's intuition about daylighting and influencing him or her to consider design elements which result in good daylighting performance. The third focuses on user satisfaction and the acceptance of the expert system by designers. More specifically, the three-phased study was designed to help answer the following questions:

- Can the expert system still improve the performance of a design when independent human interaction is included into the process?

- Can the process of working with the expert system positively influence a designer's final design, if the final design is one designed strictly by the designer and not generated by the expert system? - Can the process of working with the expert system provide a designer with increased intuition about daylighting for a specific design problem? Can it educate a designer about general daylighting concepts?

- If there is a benefit to using the expert system design process, is this benefit greater to participants who have had little to no experience working with daylighting or to participants who have had more substantial experience?

- Do the participants accept the expert system as a design tool? Would they use it for an actual design project?

The adopted approach, its originality and results are discussed in section 4.

\section{Lightsolve's overall concept}

The general approach for Lightsolve is to inform well-balanced daylight design during early design stages through an interactive visualization and a pro-active, guided improvement of fullyear time-varied daylighting performance [15]. 
The metrics used in Lightsolve differ from most existing daylighting simulation programs in two ways: they are goal-based and they place emphasis on the variation of daylight performance over the day and the year by use of temporal maps [18] (see Figure 1b).

In the Lightsolve framework, we use that representation to show how closely the users' current design fulfills their own (or standard-based) visual comfort, solar gain and light distribution goals, on an annual time-varied basis that accounts for weather conditions. An intuitive color scale (Figure 1a) indicates how closely the goals are met over the year [17]: yellow indicates that the goals were met for this sensor (or that solar gains are neither excessive nor insufficient), red indicates that values were too high, and blue that they were too low. The three Lightsolve metrics express the performance of each entire area of interest (big or small, defined by the user) rather than on a point per point basis.

Figure 1: Reading Lightsolve Temporal Maps - a) Color scale b) Correlation of time/day with performance (color) and c) with lighting distribution (renderings).

Year-representative series of renderings (Fig 1c) are also produced and associated to a given time of day/year and weather condition (the dominant one e.g.). In the Lightsolve interface (Figure $2 a)$, these renderings (right $+2 b$ ) are interactively displayed together with sky type occurrence (2a upper left) as one moves a cursor over the temporal map (middle). They are combined with the goal-based visualizations of annual performance (temporal maps) for illumination (based on desired illuminance ranges), glare (based on desired glare tolerances) and solar gains (based on probable heating/cooling needs). Their foundations and detailed mathematical implementation can be found in [17] and are briefly summarized below. 


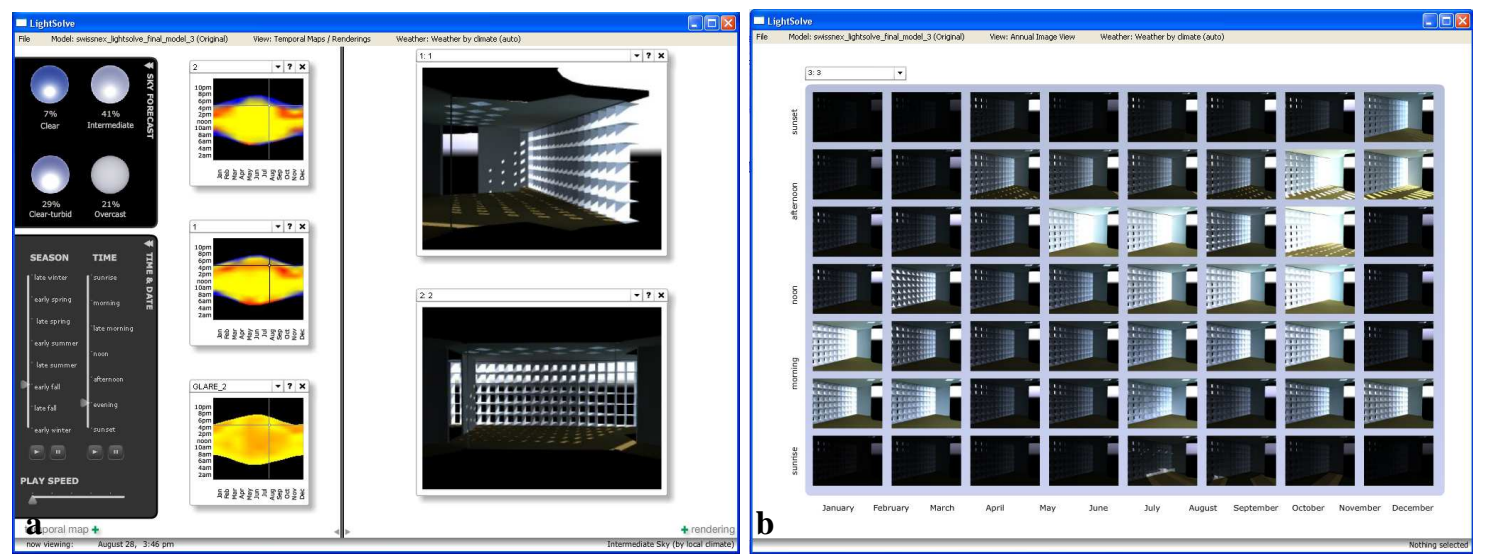

Figure 2: Lightsolve analysis interface - a) Time-varied display of performance interactively linked to renderings of the space b) Annual Image Map showing all renderings over time.

\subsection{Full year, time-varied analysis}

To allow for climate-specific outputs, illuminance and glare values are calculated for each sensor plane patch and for each of four CIE-ASRC sky types [19], ranging from overcast to clear. A climate-based representative value is then calculated as a weighted average from each sky type based on their respective occurrence during that time period. To make whole-year calculations more efficient, the year is split into 56 periods [20] and climate-based data are calculated for each of them as far as the diffuse component of daylight is concerned.

\section{Les paramètres requis sont manquants ou erronés.}

Figure 3: Condensing annual data. Museum case study a) used to compare the 56 time period reduction method with sun overlay b) against Daysim (shadow casting mode) c).

The comparisons between temporal maps produced using the data reduction method and those produced using detailed illuminance data extracted from the program Daysim at 5 minute intervals showed a strong visual and numerical correlation [20], illustrated in the example shown on Figure 3. A separate calculation for zero-bounce direct sunlight is performed for 1200 sun positions (80 times of year and 15 times of day) and results are combined to the first (diffuse) set [21].

\subsection{Rendering and calculation engine}

The overall intent of Lightsolve being to inform design in an exploratory way, there was a need for a quick calculation engine that could produce both numerical results (at the basis of temporal 
maps) and renderings over a whole year so that interactivity could be maintained. A hybrid global illumination method was developed for this purpose at the Rensselaer Polytechnic Institute, called the LightSolve Viewer or LSV [22]. It relies on patch-based radiosity for the sky and uses indirect illumination and shadow volumes for pixel-based shadows for direct illumination by the sun. This rendering system was validated through a set of qualitative and quantitative comparisons with Radiance and a pixel difference of less than $10 \%$ was found between LSV and Radiance for a variety of different scenes, camera positions, and daylighting conditions [22].

\subsection{Goal-based metrics}

Unlike most daylighting analysis tools, the three metrics that were developed for Lightsolve and are represented as colored temporal maps emphasize the time-variation of light over its detailed spatial distribution. They are also explicitly goal-based: performance objectives in daylighting can indeed vary greatly depending on the type of space and the intentions of the designer. A set of three metrics, whose underlying principles are illustrated in Figure 4, were developed to display goal-based performance information for a user-defined area of interest on a single temporal map and to offer a comprehensive and intuitive way to represent annual daylight performance of a design proposal; details can be found in [17]. A color scale - consistent amongst the three metrics - indicates how closely the goals are met over the year.

\section{a) Illumination}

The illumination metric, called Acceptable Illuminance Extent (AIE), represents the percent of an area of interest (represented by a sensor plane split into a grid of sensor patches) that stays within a user-defined illuminance goal range i.e., in essence, the amount of space which stays within acceptable limits from the standpoint of illuminance objectives. At any given moment in time, it assigns full credit to sensor patches that are within range, and partial credit (on a linear scale) to portions out of range but within a user-defined 'buffer' interval, as illustrated in Fig. 4a [17].

Because this metric condenses the spatial distribution of illuminance (over the full sensor area) into a single value (expressed in percent area), the variation of that percent over time can be displayed on a temporal map, as in the example shown in Fig. $1 b$. 


\section{b) Glare perception}

Similarly, a single number representative of overall glare perception within an area of interest is introduced as Glare Avoidance Extent (GAE) and based on the Daylight Glare Probability (DGP) metric [23]. The derived GAE metric used in Lightsolve indicates the proportion of the glare zone or glare sensor area that falls above the glare threshold considered non-acceptable by the user. It can therefore represent the glare risk for a particular location and a particular viewpoint over the year (small unique glare sensor with its normal facing that direction) but can just as well indicate the overall glare risks for a space or a range of viewing locations (e.g. for all the students in a classroom). Because this glare analysis is run annually and often for multiple viewpoints, it required more efficient methods for computing glare, that were developed for that purpose [17] and are schematically illustrated in Figure 4b. It relies on threshold values suggested by the DGP author for glare tolerance [24].

\section{c) Solar gains}

Finally, a new solar gains metric called Solar Heat Scarcity/Surplus (SHS) is used to convey the urgency of either allowing more direct solar gain or avoiding it, based on revisited balance point calculations [17] (see Fig 4c). While dynamic energy analyses should ultimately be used in determining energy loads, balance point can be as useful indicator in the earliest stages of design. The recently released 16 DOE Benchmark Commercial Buildings [25] was used to validate this approach. Within the Lightsolve environment, the Solar Heat Scarcity and Surplus metric requires additional input about the thermal properties of the envelope and building type and occupancy but is able to provide a good approximation of how much of a liability or benefit the daylight-associated solar gains are for the proposed designs.

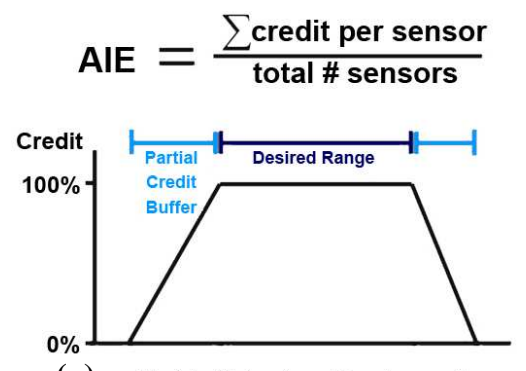

(a) Metric Value (ex. Illuminance)
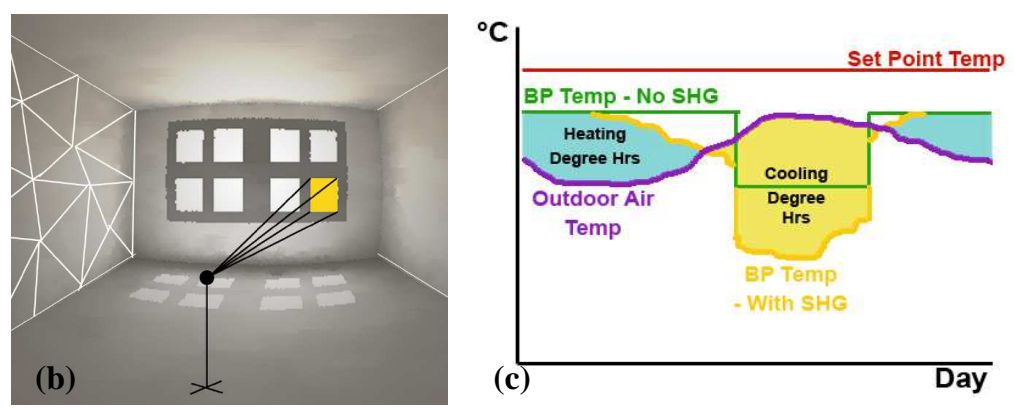

Figure 4: Lightsolve Metrics - (a) Acceptable Illuminance Extent (AIE) credit system (b) Glare Avoidance Extent (GAE) derived from DGP and based on window luminance and radiosity model (c) Solar Heat Surplus/Scarcity (SHS) based on cooling/heating daily totals. 
Although the non-spatial aspect of solar heat gain usually makes it more difficult to analyze along side illuminance or glare, the value of resorting to time-variant graphics was made evident from its ability to provide a basis of comparison so that solar heat gain information can be comparable with location-based data.

\subsection{Expert system}

Expert systems [26] offer a powerful way to introduce performance-driven decision support into the design process. The development of the Lightsolve expert system, its implementation including a user interface (Figure 5), and a brief performance assessment have been published in [16].

The expert system consists of two major components: a daylighting knowledge-base which contains information regarding the effects of a variety of design conditions on resultant daylighting performance [14], and a fuzzy rule-based decision-making logic [27] that is used to determine those design changes most likely to improve performance for a given design [16]. As in Lightsolve's analysis mode, the user is allowed to create a $3 \mathrm{~d}$ model of his/her own design and to input project-specific performance goals for illuminance and glare within the space. A user interface has been developed which displays the current performance of the design and the list of suggested design changes to the user, who is then guided towards improved performance like by this "virtual daylighting consultant". The objective is to increase annual performance for all areas of interest and all (possibly conflicting) performance objectives through the most suitable sequence of decisions - and compromises. By involving the designer and utilizing his or her knowledge as well as the intelligence built-in the tool, the process is made more efficient while the exploration space is expanded. 

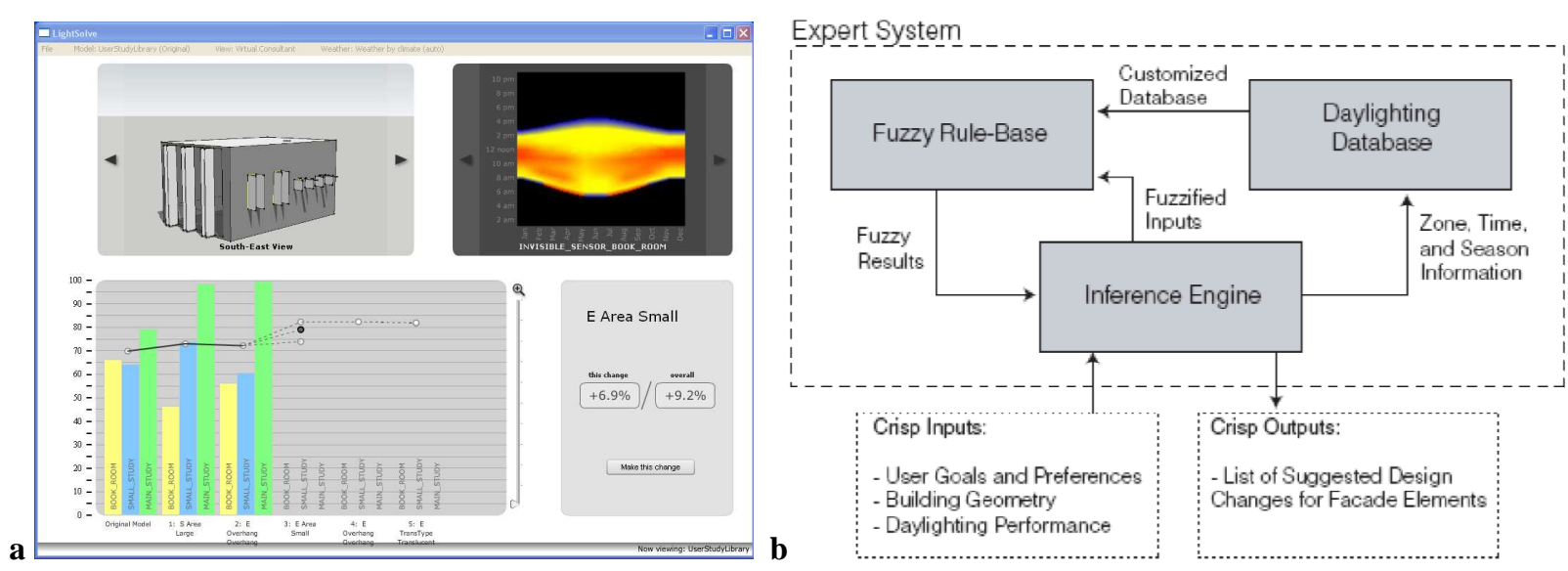

Figure 5: Lightsolve Expert System (Virtual Consultant) - (a) Guided search interface and (b) Iteration process.

\section{Preliminary user studies}

The proposed goal-based framework has by now been applied in different contexts, including research projects in Italy and Belgium [28-29], and classroom settings at MIT, UC Berkeley and EPFL in Switzerland where architecture and building technology students were encouraged to use Lightsolve for their design projects.

Two pilot user surveys were conducted at MIT early on in Lightsolve's development effort, in parallel with gathering class student feedback through questionnaires. The questionnaires focused mainly on Lightsolve's functionality and usability issues and did not lead to unexpected findings: the users mainly praised its visual and temporal approach and complained about its installation, modeling restrictions and lack of robustness. Given that these issues resulted mostly from the early development stage at which the tool was tested and are thus of little scientific interest for this paper, this section will focus on the two pilot surveys conducted in better controlled contexts. The aim of these two initial surveys was to test the usefulness of Lightsolve's time-based approach (including how intuitive the temporal maps are), and see how comfortable the architects were when interacting with this early version of the software.

\subsection{Potential for design support}


A first survey was given in January 2009 to a small number of workshop participants. Held at MIT, the 3-day workshop taught participants to use both Lightsolve and Ecotect [30] - with exports to Radiance and Daysim through a workflow similar to [7]. Participants were assigned a daylight design problem to address using both the Lightsolve program and the Ecotect-RadianceDaysim sequence (as a control), which was selected as the most advanced set of daylighting analysis tools available at the time.

The survey given at the end of the workshop focused on software usability as well as data presentation, although only the latter is relevant to this paper. An initial ambition was also to test whether participants created more successful solutions using Lightsolve's analysis format than with existing software but time was too short for the participants to go through enough design iterations of their project to enable such conclusions to be drawn; this perspective is the specific focus of the study described in section 4 .

Of the 13 people who took the Lightsolve user survey, 9 were students or practicing architects, 3 were lighting specialists and one was an engineer. None were the authors' own students or immediate colleagues. When asked how intuitive each program set was, they gave Ecotect an average of 2.86 out of 5, and Lightsolve an average of 3.55 out of 5 . When asked which program was more useful in early stage design, 10 of 13 chose Lightsolve, but when asked which was quicker to use, 8 of 13 chose Ecotect. This may have been influenced by Lightsolve's limited functionalities and usability at the time of this early prototype and drove the need for a follow-up study.

\subsection{Potential for data visualization}

As a further exploration, another survey about data presentation was given which did not depend on learning or using Lightsolve. This stand-alone survey was conducted in May 2009 with 58 MIT architecture students enrolled in master's level building technology classes; none of the students were the authors' own students or research colleagues. Through queries about software experience, and familiarity with a number of daylighting terms, participants were shown to have little daylighting experience in general.

Before the survey, a very brief introduction to workplane illuminance, temporal maps and Daylight Autonomy (i.e. the percent of occupied hours over the year where a minimum illuminance threshold is met by daylighting alone [31]) was given by the survey administrator. 
The survey was administered during class (unpaid) via strictly timed instructions and survey phasing; participants used their personal computers to take it.

The survey focused on the comparison between spatial and temporal daylighing performance outputs to judge how intuitive temporal data was to the inexperienced architect. Given a basic model of two classrooms with windows facing either Southeast or Northwest and a set of predefined daylighting goals, participants were asked to assess how well the model had achieved these goals for two design iterations and two types of visual displays (four scenarios total): results were either displayed in spatial format (falsecolor illuminance and Daylight Autonomy plots, used as a control, cf. Fig 6b) or as temporal maps (Fig. 6a) displaying Acceptable Illuminance Extent calculated over each classroom's workplane (cf. section 2.3a).

Paraphrasing, the six questions asked about each displayed scenario were:

1) did the scenario meet the goals during occupied hours?

2 ) if not, what was the biggest problem?

3) when do problems occur?

4) where in the room do problems occur?

5) how confident are you in your analysis?

6) what other information do you want?

Four follow-up questions were also asked:

Q1) which of the two data types was the most intuitive?

Q2) which of the two data types was the quickest to analyze?

Q3) which of the two data types was the most intuitive?

Q4) did you take weather into account?

Participants were given 3 minutes to answer questions about the first scenario and 2 minutes 15 seconds to answer the same questions for each of the three following scenarios. The restrictive time limits forced participants to rely on intuition and put a special emphasis on the speed of the analysis. The tallied responses to all scenarios and follow-up questions are given in Figure 7. 

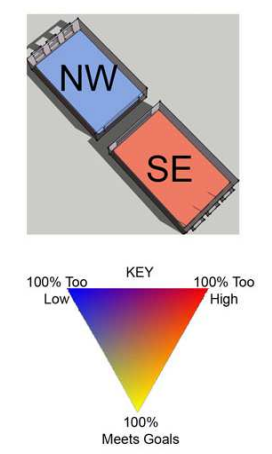

$\mathbf{a}$
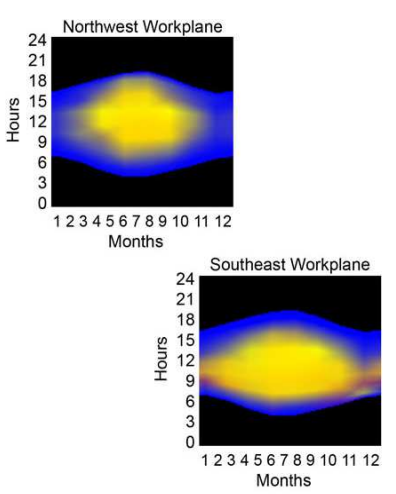

b

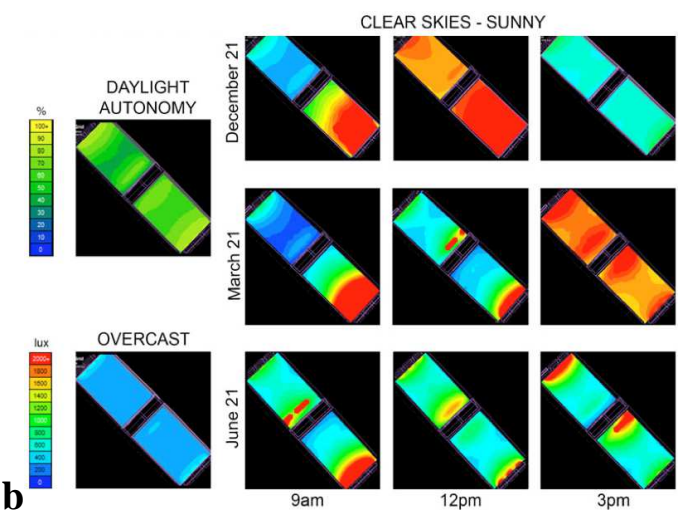

Fig 6: Typical presentation format used to compare available temporal (a) and spatial (b) data.

\subsection{Findings from preliminary studies}

The consistency in responses to the temporal data - especially questions 2 and 3 regarding what the problem was and when it happened - show that temporal maps are both readable to the untrained eye and quick, intuitive methods for displaying comprehensive daylighting data. Also, several participants commented on the "at-a-glance" nature of temporal data and the intuitive nature of the "goal-range" color scheme, although another comment unfavorably mistook the temporal maps as a collection of average illuminances. Both surveys, interestingly, revealed the architects' attachment to spatial graphics. Renderings and illuminance maps were the most requested pieces of "extra information" in question 6, and a surprising number of people revealed - through comments and confidence levels - an abiding faith in traditional single-moment illuminance graphs. The spatial data was also considered more complete, as the discrete illuminance graphs gave an illusion of temporal information. This can also be seen in the fact that nearly no one considered the spatial data "not enough information" to answer the time-dependent question 3 (and the answers were highly inconsistent with each other), but the vast majority recognized there was not enough information in temporal maps to answer the spatial-dependant question 4. Finally, for the improved model, participants decided that the biggest problem was "too much light" when given spatial data, and either no problem or "not enough light" when given temporal data. This is most likely because too much attention was paid to the clear-skies illuminance data over the climate-specific Daylight Autonomy, while the temporal maps took Boston weather (which is reasonably often overcast) into account. The majority of participants admitted that they did not take weather into account when using spatial data. 

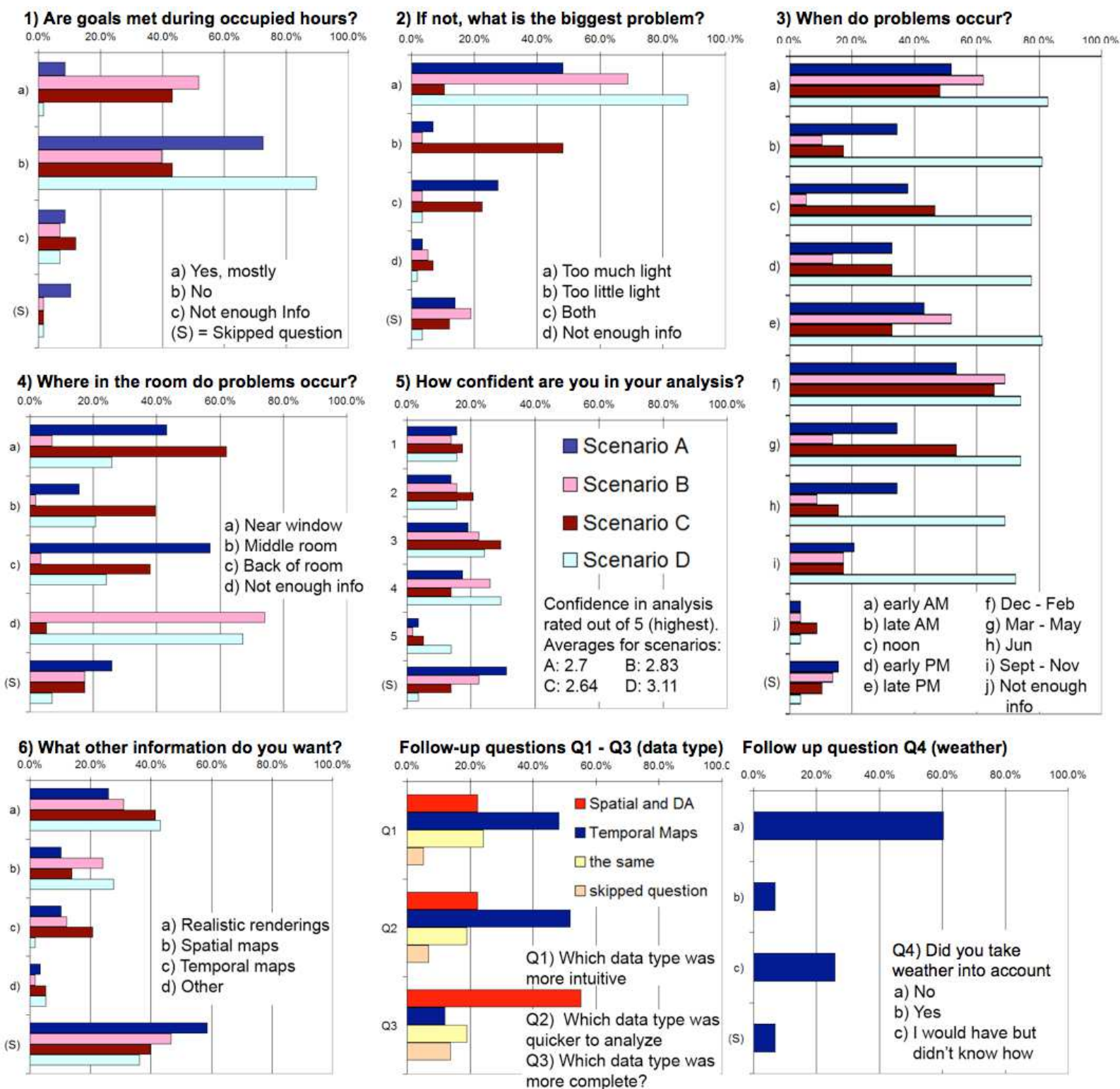

Figure 7: Survey results: Scenario A is the original model spatial data, B is the improved model temporal data, $C$ is the improved model spatial data, and $D$ is the original model temporal data.

In summary, these two pilot surveys were successful in proving the usability and usefulness of temporal daylighting data. They were however, unconclusive regarding the improvement of an architect's understanding of daylighting performance when combined with spatial data. This requires a different type of user study, focused on its added value during a design process: an approach was developed for this purpose, presented in section 4.

\section{Assessment of the expert system: an iterative user study}


A major goal of the Lightsolve project was to develop an intuitive tool and a performance-driven design process which could be used by designers to improve daylighting performance in the early design stages.

The tool was evaluated against high performing benchmark designs generated with a genetic algorithm [11] and tested for varying levels of aesthetic constraints. The results of these studies indicated that the expert system was successful at finding designs with improved performance for a variety of initial geometries and daylighting performance goals [16] when automatically accepted in the absence of user interaction. Yet because the expert system was developed to be a user-interactive tool, a critical part of this research remained to assess it when used by human designers.

To evaluate the performance of the expert system when incorporated into the design process, a user study was conducted during which designers were asked to interact with the system and solve a daylighting design problem as a three step process: first, using their own intuition, next, using the expert system, and finally, again using their own intuition. This format was designed to determine whether the use of the expert system was able to improve the daylighting performance of each participant's second and final designs when compared to his or her initial design.

\subsection{Methodology}

The aim of this user study was to identify the limitations of the expert system, without necessarily extracting a statistical behavior of the sample population. For such usability tests, a limited number of participants is typically required - in general 5 to 8 [32-33].

\section{a) Profile of Participants}

Twelve participants were ultimately included in this study (about half male and half female), all MIT architecture students and/or practicing architects between 20 and 40 years old (about half at $\mathrm{PhD}$ level, the other half at Master's level, plus one design faculty member) recruited through a posting on the general MIT architecture email list. Participation was compensated at a rate of 
$\$ 15 / \mathrm{h}$ for the 2 hours of the study, and all sessions were conducted in the same, windowless room at MIT. Because the goal of the study was to evaluate the expert system for use by designers, only those with at least one prior degree in architecture and with at least one year of experience working for an architectural design or architectural consulting firm were allowed to participate. The median work experience in a design firm was 3.8 years (minimum 1 year, maximum 8 years). Of the twelve participants, four had previously completed a bachelor's level degree in architecture and eight had completed a master's level degree in architecture. Additionally, most participants were in the process of completing a second or third degree in architecture or a related field at the time of the study.

The participants were also selected so as to represent a variety of backgrounds in daylighting. They were asked to rate their experience level in working with daylighting using one of 4 categories: experienced, intermediate, novice, or none. The group was fairly evenly split, with four (self-ranked) experienced daylighters, three intermediates, and five novices; two participants were master's students of the same research group as the authors' (i.e. focusing on daylighting), though working on topics unrelated to Lightsolve. No participant chose "no daylighting experience".

\section{b) Study Procedure}

The user study was conducted as a series of brief segments, which included three design sessions and two questionnaires. The total amount of time used for each participant was approximately two hours. The design problem will be described in detail in the next section. The same design problem was solved by each participant three times: first by hand, then using the expert system, and finally by hand again. During all sessions, participants were allowed access to pencils, blank paper, a calculator, and a stereographic sun course diagram for Boston, MA.

The sessions of the study were organized as follows:

- Introductory Questionnaire (basic information on design and daylighting background)

- General Tutorial (including task description and brief explanation on daylighting metrics)

- Design Session \#1 (design problem to complete by hand from an initial massing model and daylighting performance goals)

- Design Session \#2 (same design problem using only the expert system)

- Design Session \#3 (final version of design) 
- Final Questionnaire (satisfaction with the final design, experience of using the expert system, use the tool in a real design context), provided in full in the appendix.

The completion of questionnaires and the drawings "by hand" were all done on paper; design session \#2 was conducted on the computer made available for the survey.

In Design Session \#1, the participant was asked to design two façades on the massing model and attempt to meet the daylighting performance goals as well as satisfy him- or herself as a designer. An example of a previous façade design was provided, along with the performance of that example design (Figure 8a). During this design session, the participant was asked to sketch his or her design by hand and to draw the final design on a template sheet.

In Design Session \#2, the participant began with the same example model and was allowed to choose to accept or decline design changes suggested by the expert system, to choose the magnitude of the design change, and to return to previous design iterations. The participant was also allowed to explore designs which resulted in decreased performance if desired. During this session, the participant was not allowed to change the design by hand or in SketchUp.

In Design Session \#3, the participant was told that this design would be considered "final". He or she could revisit either or both of the designs produced during the first two sessions or completely start over.

The purpose of the three design session format was to determine if the process of using the expert system was able to positively influence each participant's final design. To create an initial design during the first design session, participants relied primarily on their own intuition and understanding of daylighting. Participants were not told how well these initial designs performed based on the daylighting goals. During the second session, participants all worked with the same starting design, which in many cases was quite different from their own initial design. During this session, however, participants were able to view the performance of the model after they applied various changes to it.

One hypothesis of this study was that if participants chose design changes that resulted in improved performance during the expert system session, they might elect to apply some of those design changes to their designs during the third session. A corollary of this hypothesis was that if participants made these design changes to their own initial designs, the performance of those designs should improve. This improved performance would indicate that participants were able to learn something about working with daylighting by using the expert system, and that the process 
of using the expert system, even for a seemingly unrelated design but for the same space characteristics and goals, could influence participants to incorporate certain design elements into their own designs.

\section{c) Design Problem}

The participants were asked to work through a conceptual design for the façade of a school library wing in Boston, MA which should use natural light instead of artificial light as much as possible. This design problem was developed to be of medium level difficulty so that designers who were experienced with daylighting concepts could create a very good solution using only their intuition. The problem was meant to be challenging for those designers who were not experienced with daylighting, but not so difficult as to discourage them.

Participants were informed that they were taking over the project from a colleague who had already started working on the design. They were required to keep the original massing model that their colleague had designed (footprint, wall heights, and interior walls). However, they were allowed to change the façade elements as necessary to meet the daylighting goals. They were allowed to choose the size and placement of windows, the types of glass used, and the types, size, and placement of shading devices. The two façades that were considered were those oriented towards South and East.

The library space has three main areas: a double-height main study area, which should receive abundant light; a smaller study area that overlooks the main study area, which should receive an adequate amount of light; a rare book room, in which light must be carefully controlled. In each area, an illuminance sensor plane was modeled at work plane height. The original design and the location of sensors within the space are shown in Figure 8. Participants were told that, based on the client's description of the space, their firm had decided that the specific daylighting goals they should work towards were:

- Main Study Space: min 500 lux is desired, down to 400 lux is acceptable; no max.

- Small Study Space: min 200 lux is desired, down to 0 lux is acceptable; max 800 lux is desired, up to 1000 lux is acceptable.

- Rare Book Room: no min; max 200 lux is desired, up to 400 lux is acceptable. 

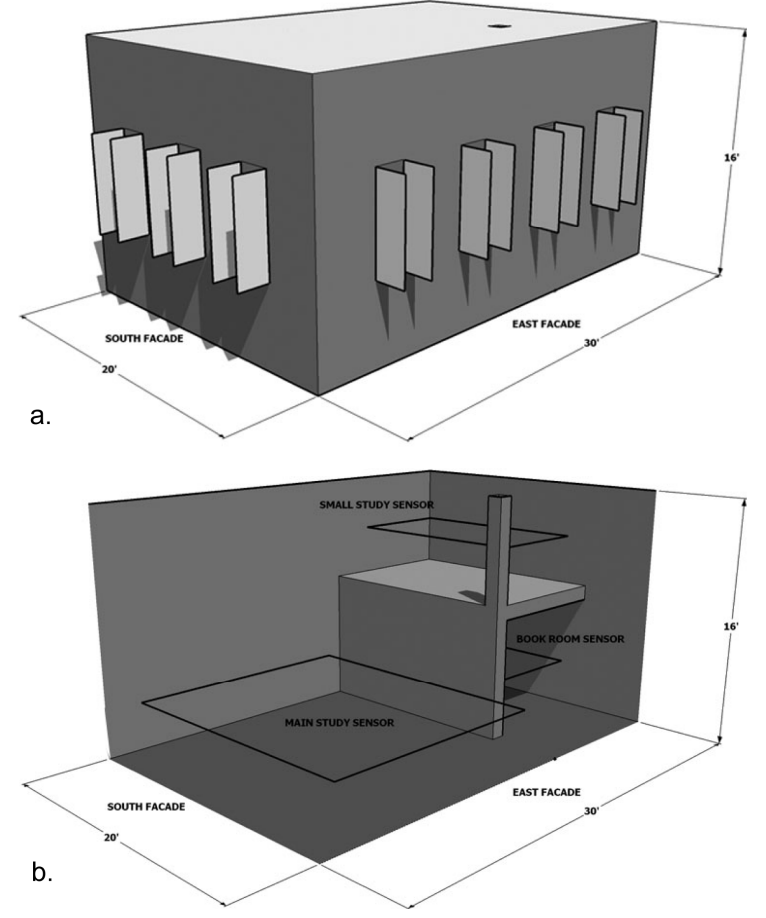

Figure 8. Library massing model with (a) South and East example façades and (b) three considered areas

Participants were told that if the illuminance on the entire area of a sensor plane falls within the desired range during all daylit times of the year, the performance of that sensor would be $100 \%$. Participants were also provided the average performances of each sensor in the example design:

- Main Study Space: $80 \%$

- Small Study Space: $65 \%$

- Rare Book Room: 70\%

- Average of All Spaces: $72 \%$

Finally, the participants were informed that the client had requested a certain aesthetic which must be maintained. The following design rules were given:

- Windows must be rectangular or square.

- Glass may be transparent or translucent.

- Glass may not be tinted with color.

- Shading devices must be opaque, and must be vertical or horizontal.

- Both vertical and horizontal shading devices may be used on the same window.

- No advanced systems, such as light-redirecting or responsive systems, may be used. 
- It is up to the designer to determine if a uniform façade aesthetic should be maintained.

- The design should achieve the daylighting goals and also satisfy the designer.

\section{d) Procedure for Modeling Designs}

For this study, it was necessary to determine the performance of models from all three design sessions, including those which the participants completed by hand.

Following each participant's study session, the authors constructed SketchUp models of the participant's initial and final designs and calculated the performance on all sensor planes using the LightSolve Viewer (LSV), that was specifically developed as an interactive daylight rendering engine [22].

The dimensions and locations of windows and shading devices on each façade were modeled based on the template sheet drawings. An example template sheet and the corresponding SketchUp model are shown in Figures 9 and 10, respectively.

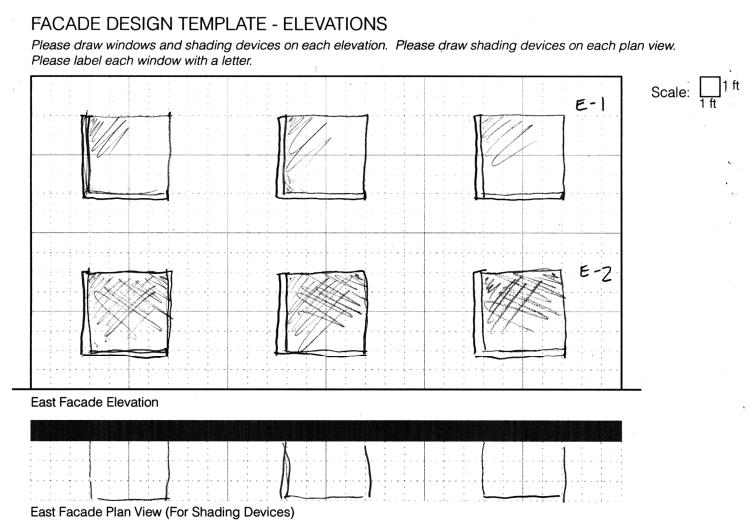

Figure 9 Example template sheet with façades drawn

For glazing types, participants were allowed to check one option in each of two categories, "View" and "Amount of Light Let In" as described below:

- View: transparent (all specular transmittance), translucent/frosty (equal specular and diffuse transmittance), opalescent (diffuse only).

- Amount of Light Let In: most (e.g. single-glazed clear, $\tau=80 \%$ ), intermediate (e.g. doubleglazed low-e, $\tau=60 \%$ ), least (e.g. neutral tint, $\tau=40 \%$ ). 
If a participant selected "translucent/frosty" and "intermediate" for instance, the glazing would be modeled as $30 \%$ specular transmissivity and $30 \%$ diffuse transmissivity.

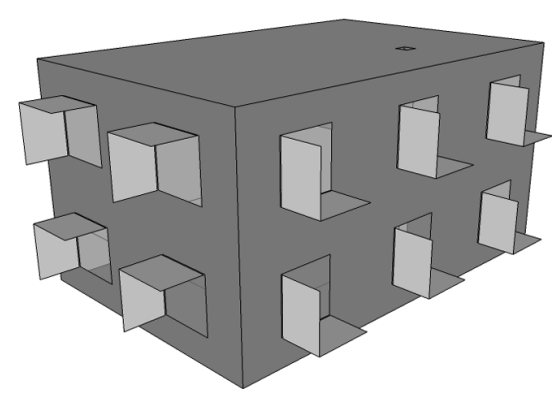

Figure 10. SketchUp model based on façade drawings

\subsection{Results}

\section{a) Performance of Designs}

To determine how closely each design model achieved the prescribed goals during each of the three design sessions, daylighting performance was estimated as follows: performance was calculated as the percentage of the total area of each sensor plane (where illuminance was calculated based on LSV renderings) falling within the desired goal range, averaged over the whole year (single value). A design which met all goals would be one for which this average value would equal $100 \%$ though the authors were not able to create a design solution that would reach this theoretical limit (98\% was the maximum performance achieved).

Performances for designs produced during each of the three design sessions are shown for all twelve participants in Figure 11. Throughout the study, each participant also had access to an example design and its performance; for reference, the performance of this example design is indicated in Figure 11 as a dashed line. The results have been ordered from least successful to most successful in terms of average whole-year performance across all three illuminance goals for the first design session. A t-test has been applied to the dataset for indicative purposes only, given the small population size: it revealed that the performance outcomes of Session 1 seemed to be statistically different from the outcomes of both Session $2(p=0.03)$ or Session $3(p=0.05)$ whereas outcomes of Sessions 2 and 3 were not statistically different $(\mathrm{p}=0.17)$. 


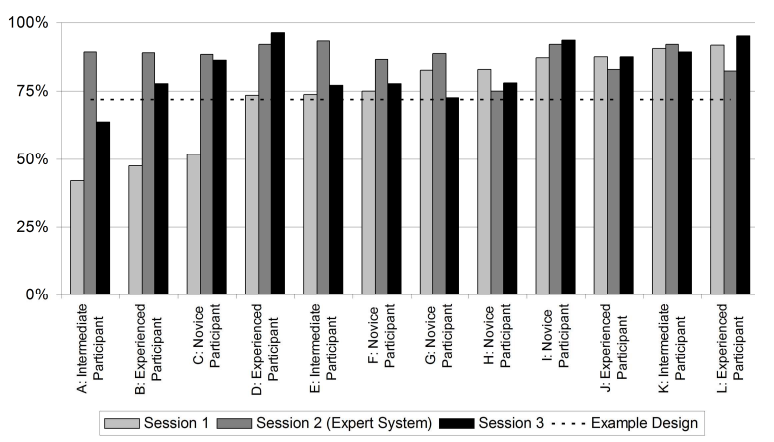

Figure 11. Performance of all produced designs (example design performance shown as dashed line)

During the first session, participants were asked to complete the design problem by hand, using only their intuition. The mean performance of the twelve designs produced during the first session was $73.9 \%$, which is close to the performance of the example design shown to each participant (71.9\%). Six participants produced designs that performed at least $10 \%$ above the example, three participants produced designs that performed similarly to the example, and three participants produced designs that performed well below the example.

During the second design session, participants were asked to use the expert system for a fixed amount of time, starting with the example model with a performance of $71.9 \%$. In general, most participants were able to make four design iterations during the allotted time (one participant was able to make five iterations and one was only able to make three iterations). The mean performance of the designs produced during this session was $87.6 \%$, and the performances of all twelve final designs were higher than that of the example model, which indicates that every participant was able to improve the performance of the starting model by using the expert system. Additionally, eleven out of twelve designs were improved by $10 \%$ or more; the design which saw the smallest improvement was created by the participant who was only able to complete three design iterations during the session.

The result that every participant was able to find a better performing design than the starting model in session \#2 is important because it demonstrates that the expert system can improve the performance of designs even when the participants' unique sets of design choices were introduced into the process. Although participants only had a short amount of time to interact with the system, and although participants were not specifically told to choose design changes which improved performance, the expert system was able to find good solutions nevertheless. 
It is also interesting to note that each participant made a unique set of design decisions and that while the final performances of some models were similar, no two participants ended up with the same final design. One result that is evident from Figure 14 below is that the set of performances of the designs found by the expert system was more uniform than those designed strictly by the participants during the first session. Using the expert system, those participants who struggled during the first session were able to find designs which performed similarly to the designs generated by those who were successful in the first session. Additionally, 9 out of 12 of the second session designs outperformed the designs created by the same participant during the first session.

During the final design session, participants were asked to revisit the same problem for a third time and to draw their final design by hand, again using only their intuition. Participants were not restricted and were allowed to draw inspiration from either or both of the first two design sessions. They could also completely start over if desired. It was the hope of the authors that during the final session participants would combine their initial design with elements from the expert system design to create a better performing final design.

The mean performance of the final twelve designs was $82.9 \%$, which was $9.0 \%$ higher than the mean performance of the initial set of designs. Additionally, nine out of twelve designers were able to produce final designs which performed the same or better than their initial designs. This improvement was particularly evident for those designers who had the least performing designs from the first session. These results are interesting because participants completed both the first and third sessions by hand, with no performance feedback. During the second session, participants did not work with their own initial design, but instead with an example design that may have had little in common aesthetically with their own initial design. This result indicates that the process of using the expert system, even with a generic example instead of their own design, was able to improve the intuition of some of the designers about ways in which performance could be increased.

As far as the comparison of results between participants with varying levels of daylighting expertise goes, one initial hypothesis was that participants with little to no previous daylighting experience would benefit more from the process of using the expert system than participants who had more substantial previous experience working with daylighting. The results of this study instead indicate that those who benefited the most from the process of using the expert system 
were those who produced the least successful initial designs, and that these participants did not necessarily consider themselves inexperienced at working with daylighting.

Based on the performance of their initial designs, the participants can be divided into three general groups: Group A consists of three participants whose initial designs performed worse (more than 20\% lower) than the example design, Group B consists of three participants whose initial designs performed about the same as the example (within 3.5\%), and Group C consists of six participants whose initial designs performed better than the example by $10 \%$ or higher. The performances of each of the three groups are shown in Figure 12.

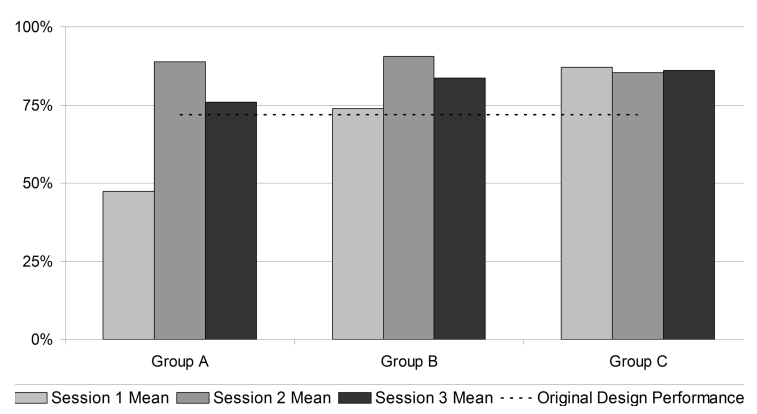

Figure 12 Mean performance for groups $A, B$, and $C$

Figure 12 shows clearly that the greatest benefits from using the expert system occurred for those in Group A, the participants whose initial designs performed the least successfully. These results are intuitive as there was more room for improvement if participants began with a lower performing design than if they began with a high performing design. However, these results also demonstrate that the expert system allowed those participants who produced the weakest initial designs to ultimately produce designs whose performance approached those developed by the more successful designers. While the difference in mean performance between Groups A and C was close to $40 \%$ for the initial designs, the process of using the expert system reduced this difference to only $10 \%$ for the final designs.

Additionally, the results show that the mean performance of the Group A final designs was about the same as the mean performance of the Group B initial designs, and likewise, the mean performance of the Group B final designs was about the same as the mean performance of the Group C initial designs. Such results indicate that the process of working with the expert system 
between the first and third design sessions effectively allowed participants in the lower two groups to "move up" one group.

It is interesting to note that Groups A and B each consisted of one self-rated novice, one intermediate and one experienced daylighter. Group C consisted of three novices, one intermediate, and 2 experienced daylighters. For this particular study, the self-rated experience level of each participant had little to no correlation with his/her initial design' performance.

\section{b) Qualitative Results}

In addition to quantitative results based on design performance, the user study produced qualitative results, based primarily on the participants' responses on the final questionnaire (provided in the appendix) and observed behavior of participants during the study. This section includes these qualitative results as well as a brief analysis of the influence of the expert system on the aesthetics of each participant's final design.

To assess how the expert system might influence design, the first 2 questions on the final questionnaire asked the participants how they felt about their final design as compared to their first design, when they considered performance and aesthetics. These two questions were designed to help determine how the process of using the expert system during the second design session influenced each participant's final design. The participants' responses to these questions are shown in Figure 13a. Each participant was also asked directly if the process of using the expert system influenced his or her final design (Figure 13b).

From Figure 13b, one can note that eleven out of the twelve participants felt that they were at least somewhat influenced by the process of using the expert system, and that four participants were greatly influenced. Figure 13a indicates that, in general, the participants felt that their final designs performed better or the same as their initial designs, but that the aesthetics of their final designs were the same or worse than their initial designs. These responses indicate that in many cases, the expert system may have influenced the designers to sacrifice aesthetics in some way for performance, despite the fact that they were told during each design session that they were supposed to try to meet the daylighting goals and to satisfy themselves as a designer. 

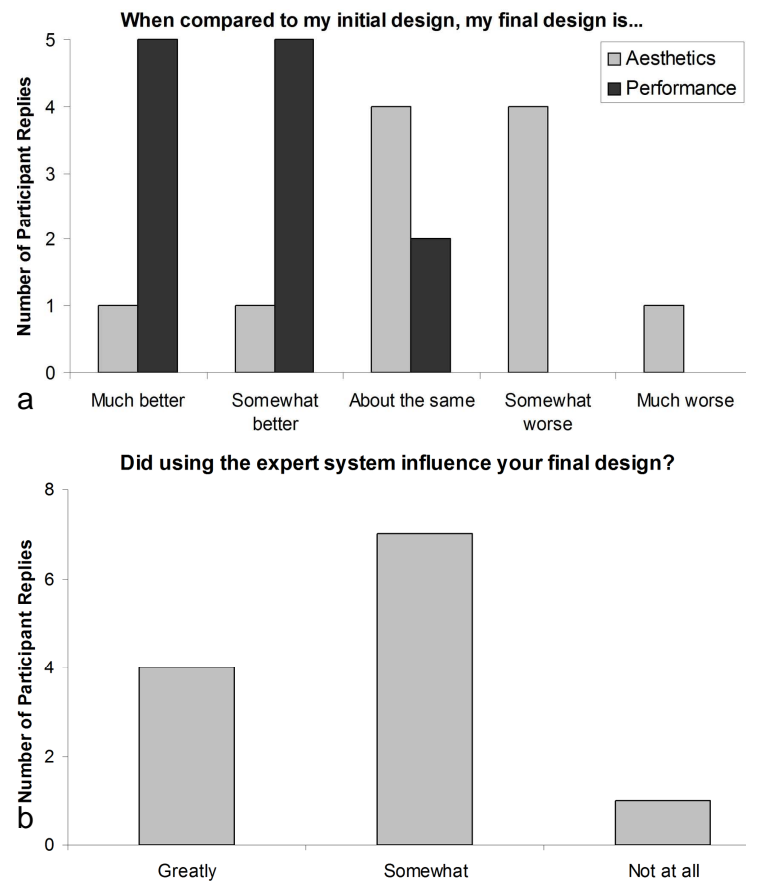

Figure 13 Participants responses about final design regarding aesthetics (a) and design impact (b)

A visual examination of the designs produced by the participants over the course of the study also makes apparent the influence of the expert system on the final designs. In many cases, the final design is aesthetically derivative of both the participant's initial design and the design produced during the expert system session. Two example sets of such designs are shown in Figure 14. In both sets, the participant combined elements from his or her original with elements from the expert system design to create a final design.

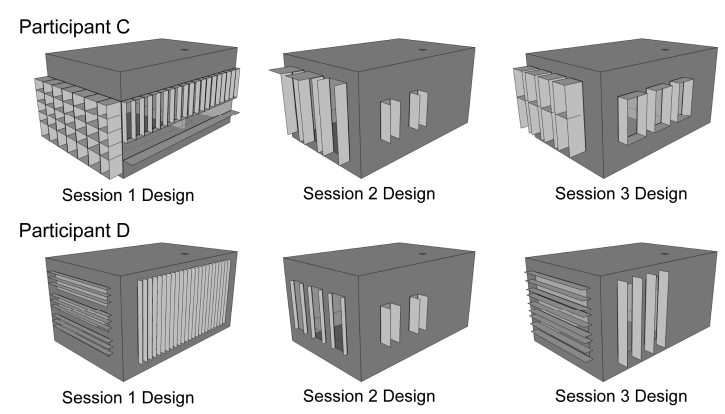

Figure 14 Two sets of designs from all three sessions 
In terms of educational value, it was shown using quantitative data (see above) that the process of using the expert system helped many of the participants improve their designs, particularly those whose initial design did not perform successfully. Participants were also asked two questions on the final questionnaire about the educational value of using the expert system: first, whether they thought that they learned something new which helped them approach the specific design problem, and second, whether they thought that they learned something new about daylighting in general. The responses to these questions are shown in Figure 15.

The majority of participants responded that they learned a "small amount" about both the specific design problem and about daylighting in general, a positive result. That they learned a "small" rather than a "large" amount may be partially due to having only a limited amount of time (40 min) to work with the tool, or to not having offered a more moderate option (like "fair amount" e.g.). It is interesting to note that although two participants claimed that they did not learn anything new about the design problem by using the expert system, these 2 participants were those who saw the highest improvement between their initial and final designs $(30.2 \%$ and $34.4 \%)$.

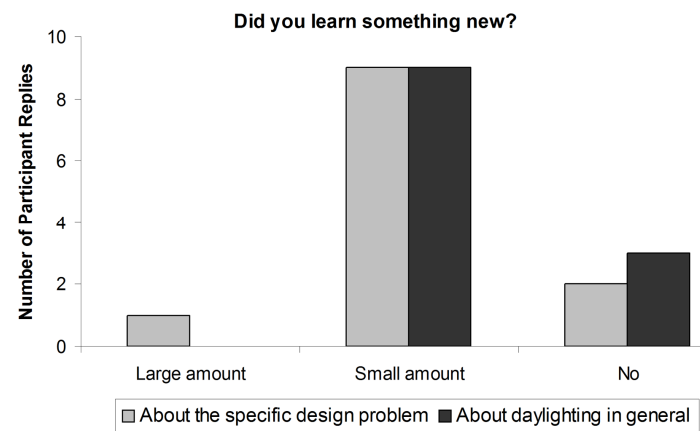

Figure 15 Participants responses on educational value

In terms of assessing how the expert system can be used to support design, participants were asked two questions on the final questionnaire to help determine whether they were satisfied with the expert system as a design tool: first, whether they would consider using the expert system again for a studio project, and second, whether they would consider using the expert system again for a professional design project. The participants' responses to these questions are shown in Figure 16. 
For both studio and professional projects, about two-thirds of the participants responded that they would consider using the expert system. Given the limited amount of time and control that participants had in using the expert system during the study, this is a positive result. There were no participants who responded that they would "definitely not" use the tool for a future project, and only two participants replied that they would "probably not" use the tool for a professional project. In general, the responses to these questions indicated mostly positive reception of the expert system.

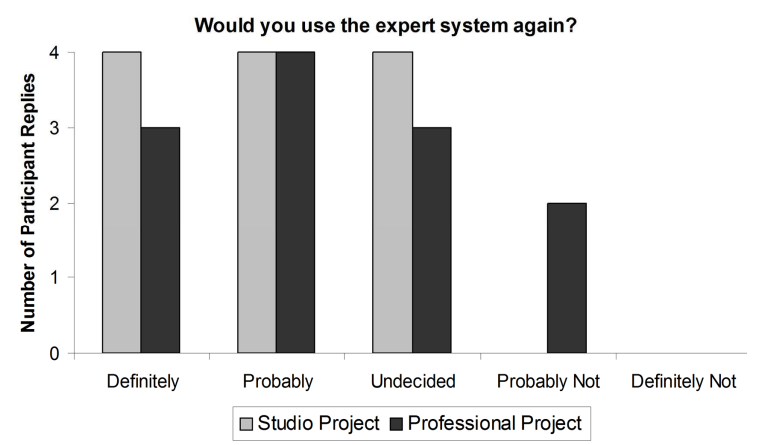

Figure 16 Participants responses on using the system

\section{Conclusions}

This paper discusses a new approach in daylighting simulation named Lightsolve from the user perspective. The main innovations are pointed out and brought together in a holistic overview of the project, that shows Lightsolve's potential as a complementary method to daylighting performance evaluation: instead of summarizing time and emphasizing spatial light distribution, it offers a way to evaluate broader areas in a space with an emphasis on how this performance varies over the seasons and time of day.

Several user studies were initially conducted to assess its adequacy as a design support tool. These pilot studies revealed that Lightsolve's goal-based color-scale and temporal maps were both very intuitive representations of performance. The main challenge is that they require users to make performance goals explicit, an unusual exercise for designers despite the fact that the evaluation of analysis outcomes is in essence always done against some kind of objectives (whether formulated or not). The studies, however, further confirmed that Lightsolve's timevaried, comprehensive goal-based metrics had a significant potential for interactivity in the early 
stages of design and could be powerful complements to the more traditional spatial grids and annual summaries.

As its core focus, the paper presents an evaluation of the expert system on one hand as a userinteractive method for performance-driven exploration and on the other hand as a design tool, based on the results of an iterative user study. Its format proved to be very appropriate to analyze an expert system's impact on the design process. During the user study, twelve designers were asked to solve a design problem with multiple daylighting goals (set for them to allow comparisons), first using their own intuition, and second, using the expert system. The designers were then asked to solve the design problem a third time, again using their own intuition. This study procedure was developed to discover if the expert system had positively influenced the performance and aesthetics of the final designs, as compared to the initial designs. The study participants were also asked to fill out a questionnaire that allowed them to assess their own designs and their experience using the expert system.

The results of the user study were generally positive and indicate that many of the major goals of the expert system as a user-interactive tool were met. One important result was that every participant was able to find a design with improved performance during his or her session with the expert system, independently of his or her self-rated daylighting expertise (which had surprisingly - no correlation with the actual performance of the proposed designs). While the [16] paper verified that the expert system could successfully work towards improved designs in the absence of a human user (similarly to traditional optimization like Genetic Algorithms e.g., as in [11]), this paper demonstrated that the expert system algorithm is also successful when human input is included in the process.

Another important result of the user study was that many of the participants were positively influenced by the process of using the expert system. Most participants also seem to have learned something about the specific design problem, which allowed them to intuitively develop better performing designs after they had interacted with the expert system. These results were supported by both the data and by participant response. A final important result is that the majority of the participants responded that they would use the expert system again for a studio or professional design project.

One possible limitation of the user study is the short amount of time that each participant was able to spend designing and interacting with the expert system. Because the sessions were 
restricted to a maximum of two hours, the designers may not have been able to respond as creatively as they may have been with more time. In many design situations, the designer is allowed to assess and redesign many times before selecting a final design. On the other hand, the fact that annual renderings could not be generated for time-efficiency reasons was another limitation imposed by this exercise, even though Lightsolve, as a tool, actually makes their simultaneous visualization with performance a priority (see Fig. 2): qualitative aesthetic considerations of course play a key role in driving decisions regarding daylighting in more realistic design contexts. Therefore, some of the behavior observed during the study may not have been indicative of how participants would have acted in a less formal and less timeconstrained environment. Additionally, the number of participants involved in the study was relatively small. Nevertheless, the study was able to provide a glimpse at the nature of the human design process and how human designers might respond to a tool such as the expert system.

An unexpected outcome of the study was that no two participants used the expert system in exactly the same way, i.e. all participants made different sets of decisions. One consequence of this behavior was that the final design found by each participant was unique. This result was somewhat surprising because the authors assumed that some of the novice users might use the expert system as an optimization method rather than as a design tool by choosing only the first design suggestion given at each step and by always accepting the best performing option. Instead, however, it was found that all participants had strong opinions of their own about which design changes to try and about how their final design should look. This type of behavior supports the idea that many designers would not readily accept a design solution generated by a "black box" algorithm. Instead, the highly interactive nature of the expert system allowed each participant to remain actively involved in the expert system design process by retaining control over design decisions. It is the belief of the authors that the participants' mostly positive receptions to the expert system were due to the interactive nature of the expert system tool.

The ultimate aim of a simulation platform like Lightsolve is to allow a user to address and evaluate the numerous goals and constraints of a complex design scenario without negatively overwhelming him or her. In the proposed approach, given that the designer is not passively observing the production of a set of solutions but is actively involved in their generation, (s)he has the opportunity to gain a better understanding of how daylighting performance relates to 
design decisions and environmental factors, and how some decisions have more pronounced effects than others already at early design stages.

The positive feedback received during the expert system user study encourages the authors to pursue this development effort into a tool that could be used more broadly [34]. Such an interactive platform could empower the designer with a computational framework that builds upon his or her own intuition and experience - the two most cited factors in driving the design process for daylighting performance [5,35] - and become an asset in the architect's toolset.

\section{Acknowledgement}

The authors were supported by the Massachusetts Institute of Technology and would like to acknowledge additional support from the Ecole Polytechnique Fédérale de Lausanne (Prof. M. Andersen) and the Martin Family Society for Sustainability (Dr. J.M.L. Gagne and Dr. S. Kleindienst). They would also like to thank Prof. Les Norford for his insightful advice on the expert system study.

\section{Appendix: Final Questionnaire administered for the iterative user study}

1. Given the aesthetic constraints, when compared to my first design, I feel that my final design is... _ Much more aesthetically pleasing

_- Somewhat more aesthetically pleasing

_ About the same

__ Somewhat less aesthetically pleasing

__ Much less aesthetically pleasing

Comments:

2. Given the daylighting goals, when compared to my first design, I feel that my final design performs...

_ Much better

- Somewhat better

_- About the same

_ Somewhat worse

- Much worse

Comments:

3. I feel that the process of using the expert system...

_ Greatly influenced my final design

_ Somewhat influenced my final design

_ Did not influence my final design

Comments:

4. Do you feel that by using the expert system, you learned something new which helped you approach the 
design problem?

Yes, I learned a large amount.

_ Yes, I learned a small amount.

_ No, I did not learn anything new.

Comments:

5. Do you feel that by using the expert system, you learned something new about daylighting in general?

- Yes, I learned a large amount.

_ Yes, I learned a small amount.

_. No, I did not learn anything new.

Comments:

6. Would you consider using the expert system for a studio project? Please explain your answer.

_ Definitely

_ Probably

_ Undecided

_. Probably not

__ Definitely not

Please explain:

7. Would you consider using the expert system for a professional project? Please explain your answer.

_ Definitely

_ Probably

_ Undecided

_- Probably not

__ Definitely not

Please explain:

8. What did you like about the expert system?

9. What did you dislike about the expert system?

10. Any additional comments?

\section{References}

[1] Swiss Federal Office of Energy. 2009. Energieforschung 2009 - Ueberblicksberichte. Swiss Confederation.

[2] US Department of Energy: "Buildings Energy Data Book" (September 2007), sec. 1.3.3.

[3] Bodart M., De Herde A. 2002. Global energy savings in offices buildings by the use of daylighting. Energy and Buildings 34(5), 421-429.

[4] Ihm P., Nemri A., Krarti M. 2009. Estimation of lighting energy savings from daylighting. Building and Environment 44 (3), pp. 509-514.

[5] Reinhart C, Fitz A. 2006. Findings from a survey on the current use of daylight simulations in building design. Energy and Buildings 38 (7), 824-835.

[6] Sarawgi T. 2006. Survey on the use of lighting design software in architecture and interior design undergraduate education. International Journal of Architectural Computing 4(4): pp. 91-108.

[7] Reinhart, C.F., Wienold, J. 2011. The Daylighting Dashboard - A Simulation-Based Design Analysis for Daylit Spaces, Building and Environment, 46(2), pp. 386-396. 
[8] Mardaljevic, J., Heschong, L., Lee, E. 2009. Daylight metrics and energy savings, Lighting Research and Technology, 41(3), pp. 261-283.

[9] Marks J, Andalman B, Beardsley PA, et al. 1997. Design galleries: A general approach to setting parameters for computer graphics and animation, In Proceedings of SIGGRAPH 97, Computer Graphics Proceedings, Annual Conference Series, Los Angeles.

[10] Caldas L., Norford LK. 2002. A design optimization tool based on a genetic algorithm, Automation in Construction 11: 173-184.

[11] Gagne J.M.L., Andersen M. 2012. A Generative Façade Design Method Based on Daylighting Performance Goals, Journal of Building Performance Simulation, 5 (3): 141-154.

[12] Ochoa C., Capeluto I. 2009. Advice tool for early design stages of intelligent façades based on energy and visual comfort approach, Energy and Buildings 41(5): 480-488.

[13] Paule B., Flourentzou F., Pantet S., Boutillier J. 2011. DIAL+suite: a complete, but simple, suite of tools to optimize the global performance of buildings openings, In Proc. CISBAT, Lausanne.

[14] Gagne J.M.L., Andersen M. 2011. A daylighting knowledge-base for performance-driven facade design exploration, LEUKOS 8(2): 93-101, 2011.

[15] Andersen M., Kleindienst S., Yi L., Lee J., Bodart M., Cutler B. 2008. An intuitive daylighting performance analysis and optimization approach, Building Research \& Information 36(6): 593-607.

[16] Gagne J.M.L., Andersen M., Norford L.K. 2011. An Interactive Expert System for Daylighting Design Exploration, Building and Environment 46(11): 2351-2364, 2011.

[17] Kleindienst S., Andersen M. 2012. Comprehensive Annual Daylight Design through a GoalBased Approach, Building Research and Information 40(2): 154-173.

[18] Mardaljevic, J. 2004. Spatio-temporal dynamics of solar shading for a parametrically defined roof system, Energy and Buildings 36, 815-823.

[19] Perez R, Michalsky J, Seals R. 1992. Modelling Sky Luminance Angular distribution for real sky conditions; Experimental evaluation of existing algorithms, Journal of the IES 21(2), pp. 84-92.

[20] Kleindienst, S., Bodart, M., Andersen, M. 2008. Graphical Representation of Climate-Based Daylight Performance to Support Architectural Design, LEUKOS, 5(1), pp. 39-61.

[21]Kleindienst, S. 2010. Time-Varied Daylighting Performance to Enable a Goal-Driven Design Process, $\mathrm{PhD}$ thesis, MIT.

[22] Cutler B., Martin S., Sheng Y., Glaser D., Andersen M. 2008. Interactive Selection of Optimal Fenestration Materials for Schematic Architectural Daylighting Design, Automation in Construction 17 (7): 809-823.

[23] Wienold J., Christoffersen J. 2006. Evaluation methods and development of a new glare prediction model for daylight environments with the use of CCD cameras. Energy and Buildings 38: 743-757

[24] Wienold J. 2009. Dynamic daylight glare evaluation. Proceedings of IBPSA 2009, Glasgow, July 27-30

[25] Torcellini P., Deru M., Crawley D.B. et al. 2009. DOE Commercial Building Benchmark Models, Proc. 2008 ACEEE Summer Study.

[26] Luger G. 2004. Artificial Intelligence: structures \& strategies for complex problem solving, Add.-W.

[27] Siler W., Buckley J. 2005. Fuzzy expert systems and fuzzy reasoning. John Wiley \& Sons.

[28] Pellegrino A., Lo Verso VRM., Cammarano S. 2011. Limits and Potentials of Different Daylighting Design Approaches Based on Dynamic Simulations, Proceedings of the CISBAT 2011 conference, Lausanne, Switzerland, Sept 14-15.

[29] Piderit B., Bodart M., Norambueno T. 2011. A Method for integrating visual comfort criteria in daylighting design of school, Proceedings of the PLEA 2011 conference on Passive and Low Energy Architecture, Louvain-la-Neuve, Belgium, July 13-15.

[30] Marsh, A. 2008. ECOTECT: An Overview. Retrieved 03 07, 2008, from Square One: http://squ1.com/products/ecotect 
[31] Reinhart, C.F., Walkenhorst, O. 2001. Validation of dynamic RADIANCE-based daylight simulations for a test office with external blinds. Energy and Buildings 33(7): 683-697

[32] Virzi, RA. 1992. Refining the test phase of usability evaluation: how many subjects is enough?, Human Factors 34 (4), pp.457-468

[33] Dumas, J. 1997. How many participants in a usability test is enough?, Common Ground, Usability Professionals Organization, 7 (October), 2-6.

[34] Andersen M., Guillemin A., Amundadottir M.L., Rockcastle S. (2013, submitted). Beyond illumination: An interactive simulation framework for non-visual and perceptual aspects of daylighting performance, Proceedings IBPSA 2013, Chambéry, France, Aug 26-30, 2013

[35] Galasiu AD., Reinhart C. 2008. Current daylighting design practice: a survey, Building Research \& Information 36(2): 159-174. 


\section{Figure captions}

Figure 1: Reading Lightsolve Temporal Maps - a) Color scale b) Correlation of timelday with performance (color) and c) with lighting distribution (renderings).

Figure 2: Lightsolve analysis interface - a) Time-varied display of performance interactively linked to renderings of the space b) Annual Image Map showing all renderings over time.

Figure 3: Condensing annual data. Museum case study a) used to compare the 56 time period reduction method with sun overlay b) against Daysim (shadow casting mode) c).

Figure 4: Lightsolve Metrics - (a) Acceptable Illuminance Extent (AIE) credit system (b) Glare Avoidance Extent (GAE) derived from DGP and based on window luminance and radiosity model (c) Solar Heat Surplus/Scarcity (SHS) based on cooling/heating daily totals.

Figure 5: Lightsolve Expert System interface (Virtual Consultant) - - (a) Guided search interface and (b) Iteration process.

Fig 6: Typical presentation format used to compare available temporal (a) and spatial (b) data.

Figure 7: Survey results: Scenario A is the original model spatial data, B is the improved model temporal data, $C$ is the improved model spatial data, and $D$ is the original model temporal data.

Figure 8. Library massing model with (a) South and East example façades and (b) three considered areas

Figure 9 Example template sheet with façades drawn

Figure 10. SketchUp model based on façade drawings

Figure 11. Performance of all produced designs (example design performance shown as dashed line)

Figure 12 Mean performance for groups $A, B$, and $C$

Figure 13 Participants responses about final design regarding aesthetics (a) and design impact (b)

Figure 14 Two sets of designs from all three sessions

Figure 15 Participants responses on educational value

Figure 16 Participants responses on using the system 